\title{
Comparison of shear banding in BMGs due to thermal-softening and free volume creation
}

\author{
LIU LongFei ${ }^{1,2 \dagger}$, DAI LanHong ${ }^{2}$, BAI YiLong ${ }^{2}$ \& KE FuJiu ${ }^{3}$ \\ ${ }^{1}$ Hunan Provincial Key Laboratory of Health Maintenance for Mechanical Equipment, Hunan University of \\ Science and Technology, Xiangtan 411201, China; \\ ${ }^{2}$ State Key Laboratory of Nonlinear Mechanics (LNM), Institute of Mechanics, Chinese Academy of Sci- \\ ences, Beijing 100190, China; \\ ${ }^{3}$ Department of Applied Physics, Beijing University of Aeronautics and Astronautics, Beijing 100088, China
}

This paper reports a comparative study of shear banding in BMGs resulting from thermal softening and free volume creation. Firstly, the effects of thermal softening and free volume creation on shear instability are discussed. It is known that thermal softening governs thermal shear banding, hence it is essentially energy related. However, compound free volume creation is the key factor to the other instability, though void-induced softening seems to be the counterpart of thermal softening. So, the driving force for shear instability owing to free volume creation is very different from the thermally assisted one. In particular, long wave perturbations are always unstable owing to compound free volume creation. Therefore, the shear instability resulting from coupled compound free volume creation and thermal softening may start more like that due to free volume creation. Also, the compound free volume creation implies a specific and intrinsic characteristic growth time of shear instability. Finally, the mature shear band width is governed by the corresponding diffusions (thermal or void diffusion) within the band. As a rough guide, the dimensionless numbers: Thermal softening related number $B$, Deborah number (denoting the relation of instability growth rate owing to compound free volume and loading time) and Lewis number (denoting the competition of different diffusions) show us their relative importance of thermal softening and free volume creation in shear banding. All these results are of particular significance in understanding the mechanism of shear banding in bulk metallic glasses (BMGs).

metallic glasses, shear bands, diffusion, instability

\section{Introduction}

Bulk metallic glasses (BMGs) have attracted large interest due to their unique physical, mechanical,

Received May 15, 2007; accepted December 30, 2007

doi: 10.1007/s11433-008-0133-3

${ }^{\dagger}$ Corresponding author (email: wangerce@yahoo.com.cn)

Supported by the Chinese Academy of Sciences under the project "Multi-Scale Complex System” (Grant No. KJCX-SW-L08), the National Natural Science Foundation of China (Grant Nos. 10725211 and 10721202), and the Doctorial Start-up Fund of Hunan University of Science and Technology (Grant No. E50840) 
and chemical properties ${ }^{[1-3]}$. Localized shear bands are particularly important in metallic glasses, because they are observed under various loadings and may limit the application of bulk metallic glasses as structural materials ${ }^{[4]}$. For a long time, there have been two types of mechanism proposed to explain shear bands in BMGs: the creation of free volume ${ }^{[5-8]}$ and thermally assisted softening ${ }^{[9,10]}$. Both have had some subtle evidence analytically, experimentally and numerically. For instance, early in the 1970s, Spaepen and his co-workers indicated that shear-induced dilatation could reach dynamic equilibrium with no temperature rise ${ }^{[5,7]}$. Pampillo and others $\frac{[11,12]}{}$ provided experimental evidence for such a structural change at shear bands.

Experimentally, Bruck, Rosakis and Johnson ${ }^{[13]}$, and Hufnagel et al. ${ }^{[14]}$ reported the dynamic compressive behavior of $\mathrm{Zr}_{41.25} \mathrm{Ti}_{13.75} \mathrm{Cu}_{12.5} \mathrm{Ni}_{10} \mathrm{Be}_{22.5}$ at strain rates of $10^{2}$ to $10^{4} \mathrm{~s}^{-1}$ by using a split Hopkinson pressure bar. A high-speed infrared thermal detector was used to determine whether adiabatic heating occurred during dynamic deformation of the metallic glass. They found that "no adiabatic heating occurs before yielding". However, they observed that "temperature increases because adiabatic heating occurs after the onset inhomogeneous deformation". Then, "temperature near the melting point may be approached within shear bands after the specimen has failed”. Recently Lewandowski and Greer ${ }^{[15]}$ showed that the temperature, over a few nanoseconds, could rise as high as a few thousand Kelvin, based on a fusible coating. However, they indicated that "nevertheless, the temperature rise does not seem to control the shear band thickness. It is important to understand the mechanisms of shear banding and associated softening because these are the principal factors limiting structural applications of bulk metallic glasses”. In the same issue, Spaepen wrote a note on this matter and raised the question: Must shear bands be hot in metallic glasses? ${ }^{[16]}$.

In numerical studies of their MD simulations, Falk and Shi ${ }^{[17]}$ demonstrated that "strain localization occurs in this system despite a lack of a measurable decrease in density". But they pointed out that "this may be an artifact of the simulation". "Density fluctuations and small system size prevent us from directly detecting density changes smaller than approximately $3 \%$. However, this does indicate that density changes in the shear band region can be quite subtle and may not be the most salient detail of the process that leads to localization". Nevertheless, "nanometer sized voids are observed to form dynamically during the localization of strain”.

Theoretically, a number of works analyzed shear instability and localization owing to free volume creation (Steif et al. 1982, Huang et al. 2002) ${ }^{[7,8]}$. However, the shear instability relevant to both thermal softening and free volume creation was reported only recently, such as by Dai et al. $(2005)^{[18]}$.

Based on these references, this paper intends to make a comparative study of the effects of thermo-softening and free volume creation on shear banding in BMGs. In accord with the analysis of the two mechanisms and their magnitudes, their relative importance at different stages of shear banding, i.e. instability, growth and mature feature, is demonstrated.

\section{Governing parameters and temporal-spatial scales}

In accord with continuum mechanics, the system of equations governing the simple shear involving thermal softening and free volume creation is as follows:

$$
\rho \frac{\partial^{2} \gamma}{\partial t^{2}}=\frac{\partial^{2} \tau}{\partial y^{2}}
$$




$$
\begin{aligned}
\frac{\partial \theta}{\partial t} & =\kappa \frac{\partial^{2} \theta}{\partial y^{2}}+\frac{K \tau}{\rho C_{v}} \frac{\partial \gamma}{\partial t}, \\
\frac{\partial \xi}{\partial t}= & D \frac{\partial^{2} \xi}{\partial y^{2}}+G(\xi, \theta, \tau), \\
\tau & =\tau(\gamma, \dot{\gamma}, \theta, \xi),
\end{aligned}
$$

where $t$ is the time, $y$ is the axis vertical to shearing, $\rho$ the density, $\gamma$ the shear strain, $\dot{\gamma}$ the shear strain rate, $\tau$ the shear stress, $\theta$ the temperature, $\xi$ the free volume fraction, $\kappa$ the thermal diffusivity, $C_{v}$ the specific heat, $K$ the fraction of plastic work converted into heat, $D$ the diffusivity of free volume and $G$ the combined rate of generation and annihilation of free volume. Actually, the equations are the momentum equation, the energy equation (thermal diffusion), the free volume diffusion and the thermo-plastic constitutive equation, respectively. In order to analyze shear instability, we define some variables based on homogeneous deformation as follows: Strain hardening $Q=(\partial \tau / \partial \gamma)$, strain rate hardening $R=(\partial \tau / \partial \dot{\gamma})$, thermal softening $P=-(\partial \tau / \partial \theta)$, free volume softening $F=-(\partial \tau / \partial \xi)$, compound creation of free volume (in accord with the definition of compound damage made by Davison and Stevens $\left.1972^{[19]}\right) G_{\xi}=(\partial G / \partial \xi)$, and other two free volume related variables $G_{\theta}=(\partial G / \partial \theta)$ and $G_{\tau}=(\partial G / \partial \tau)$. Based on the data available, hereafter, we qualitatively assume all these variables be positive, though some variables may not always be. For instance, it is reported that there is strain rate softening for strain rates greater than $3 \times 10^{3} / \mathrm{s}^{-1}$ in $\mathrm{Zr}$ metallic glasses $\frac{[13]}{}$. To facilitate the following analysis and estimation of shear banding, Table 1 lists some typical values of these parameters and variables of BMGs. It is worthy to note that the order of magnitude of free volume creation $G$ and its derivative $G_{\xi}$ is just a rough

\begin{tabular}{|c|c|c|}
\hline Property & Value & Ref. \\
\hline Density $\rho\left(\mathrm{kg} \cdot \mathrm{m}^{-3}\right)$ & $5.9 \times 10^{3}$ & Conner et al. $(2000)^{\underline{[20]}}$ \\
\hline Specific heat $c_{p}\left(\mathrm{~J} \cdot \mathrm{kg}^{-1} \cdot \mathrm{K}^{-1}\right)$ & 532, at $\theta_{g}$ & Conner et al. $(2000)^{[20]}$ \\
\hline Viscosity (kinetic) $v\left(\mathrm{~m}^{2} \cdot \mathrm{s}^{-1}\right)$ & $\begin{array}{l}\sim 10^{-3} \text {, at } 1000 \mathrm{~K} \\
\gg 10^{-3}, \theta<\theta_{g}\end{array}$ & $\begin{array}{l}\text { Conner et al. }(2000)^{[20]} \\
\text { Wang et al. }(2004)^{[\underline{[3]}}\end{array}$ \\
\hline Thermal diffusivity $\kappa\left(\mathrm{m}^{2} \cdot \mathrm{s}^{-1}\right)$ & $3.5 \times 10^{-6}$ & Conner et al. $(2000)^{[20]}$ \\
\hline Free volume diffusivity $D\left(\mathrm{~m}^{2} \cdot \mathrm{s}^{-1}\right)$ & $<10^{-16}$ & Wang et al. (2004) ${ }^{[3]}$ \\
\hline Free volume creation $G_{\xi}\left(\mathrm{s}^{-1}\right)$ & $\sim>10^{0}$ & Huang et al. (2002) $)^{[8]}$ \\
\hline Free volume $\xi$ & $\sim 10^{-2}$ & $\begin{array}{l}\text { Liu et al. (2005) } \\
\text { Huang et al. }(2002)^{[8]}\end{array}$ \\
\hline Strain at yielding $\gamma$ & $10^{-2}$ & $\begin{array}{l}\text { Conner et al. }(2000)^{\left[\frac{[20]}{}\right.} \\
\text { Liu et al. }(2005)^{[21-23]}\end{array}$ \\
\hline $\begin{array}{r}\text { Strain rate } \dot{\gamma}\left(\mathrm{s}^{-1}\right) \text { : static } \\
\text { dynamic }\end{array}$ & $\begin{array}{l}\sim<10^{-3} \\
\sim 10^{3}\end{array}$ & $\begin{array}{l}\text { Conner et al. }(2000)^{[20]} \\
\text { Liu et al. }(2005)^{[21,22]}\end{array}$ \\
\hline
\end{tabular}
estimate from references and more accurate knowledge of the function is badly needed.

Table 1 Parameters and the range of variables

Linear perturbation to the system of governing eqs. (1)-(4) gives the following characteristic equation for $\alpha^{[24]}$ :

$$
a_{4} \alpha^{4}+a_{3} \alpha^{3}+a_{2} \alpha^{2}+a_{1} \alpha+a_{0}=0 .
$$

Importantly, $\alpha$ is the reciprocal of characteristic time and describes the exponential growth (when $\alpha$ 
has a positive real part) or decay (all real parts of $\alpha$ are negative) of perturbations $\mathrm{e}^{\alpha t+\mathrm{i} k y}$, where $k$ is the wave number. For the coupled case, i.e. involving both thermal softening and free volume creation, the coefficients $a_{i}$ of eq. (5) are

$$
\begin{gathered}
a_{4}=1, \\
a_{3}=\left[\beta+\rho C_{v}\left(D k^{2}-G_{\xi}\right)+\rho C_{v} G_{\tau} F\right] / \rho C_{v}, \\
a_{2}=\left[k^{2} \omega+\rho \beta\left(D k^{2}-G_{\xi}\right)+k^{2} \rho \lambda G_{\tau} F+K \dot{\gamma} \rho G_{\theta} F\right] / \rho^{2} C_{v}, \\
a_{1}=k^{2}\left[\omega\left(D k^{2}-G_{\xi}\right)+\lambda Q k^{2}-K \tau G_{\theta} F\right] / \rho^{2} C_{v}, \\
a_{0}=k^{4} \lambda Q\left(D k^{2}-G_{\xi}\right) / \rho^{2} C_{v},
\end{gathered}
$$

where $\beta=K \dot{\gamma} P+\lambda k^{2}+R C_{v} k^{2}$ and $\omega=\lambda R k^{2}+\rho C_{v} Q-K \tau P$.

Clearly, at the short wave limit (wave number $k \rightarrow \infty$ ), uniform deformation always remains stable. This is due to the fact that its characteristic equation has the following coefficients:

$$
\begin{gathered}
a_{4}=1, \\
a_{3}=\left(\lambda+R C_{v}+\rho C_{v} D\right) k^{2} / \rho C_{v}, \\
a_{2}=\left[\lambda R+\rho\left(R C_{v}+\lambda\right) D\right] k^{2} / \rho^{2} C_{v}, \\
a_{1}=\lambda R D k^{6} / \rho^{2} C_{v}, \\
a_{0}=\lambda Q D k^{6} / \rho^{2} C_{v} .
\end{gathered}
$$

Obviously, only the last two coefficients $a_{1}$ and $a_{0}$ have the highest order $k^{6}$. Hence, $\alpha$ has a negative solution only:

$$
\alpha=-Q / R \text {. }
$$

So homogeneous deformation is stable under very short wave perturbations and the characteristic time to relax to the stable deformation is $t=1 /|\alpha|=R / Q$. Hence the relaxation time $t_{R Q}$ is an intrinsic time scale for the material under consideration. In particular, all the variables relevant to softening, such as thermal softening $P$, void softening $F$, as well as compound creation of free volume $G_{\xi}$, do not appear in the characteristic equation at the short wave limit.

Actually, the parameters and variables involved in the process imply several time and length scales. The relevant time scales are: The relaxation time $t_{R Q}=R / Q$, the external time scale $t_{\mathrm{ex}}=\gamma / \dot{\gamma}$ and the time related to compound free volume creation $t_{G}=1 / G_{\xi}$. Later, we will see that $t_{G}$ is closely related to the instability growth owing to the free volume creation. From the definitions, one can notice $t_{R Q} \sim t_{\mathrm{ex}}$. From Table $1, t_{\mathrm{ex}}=\gamma / \dot{\gamma}$ is in the order of $10^{1}$ or $10^{-5} \mathrm{~s}$ under quasi-static or dynamic loadings, respectively, and $t_{G}=1 / G_{\xi}$ is in the order of $100 \mathrm{~s}$ and may vary with strain rates. Importantly, these time scales lead to some time-related dimensionless numbers. Deborah number is the most popular and important time-related dimensionless number:

$$
D e=\frac{t_{\tau}}{t_{\mathrm{ex}}}=\frac{\text { relaxation time }}{\text { observation time }} \text {. }
$$

$D e \ll 1$ implies that one can see the asymptotic state of the intrinsic relaxation, but when $D e \gg 1$, one can see the very early stage only. Clearly, one can see the whole relaxation process, only when De 1. 
For the case under consideration, the following two Deborah numbers may be relevant to our discussions. The first is

$$
D e=\frac{t_{R Q}}{t_{\mathrm{ex}}} \sim O(1),
$$

indicating that the relaxation to stable deformation matches the external loading. The second is

$$
D e_{G}=\frac{t_{G}}{t_{\mathrm{ex}}} \sim \frac{\dot{\gamma}}{G_{\xi} \gamma} .
$$

According to the order of magnitude listed in Table $1, D e_{G}$ may be in $O(1)$ for quasi-static process and may be much greater than $O(1)$ under dynamic loading. Hence, as shown later, shear banding governed by free volume creation is preferred under static loading, but may be invisible under the dynamic one. Again, we should stress that the statement is based on the data listed in Table 1, but accurate data of $G_{\xi}$ are badly needed, otherwise the route of shear banding can hardly be clarified.

On the other hand, at a fixed time scale, for instance, the relaxation time $t_{R Q}$, there are three length scales, corresponding to three different diffusions (viscosity, heat and mass). They are $l_{v}^{2}=\frac{v R}{Q} \gg l_{\kappa}^{2}=\frac{\kappa R}{Q} \gg l_{D}^{2}=\frac{D R}{Q}$, since generally the kinetic viscosity $v>$ heat diffusion $\kappa>$ free volume diffusion $D$, seen in Table 1. Incidentally, the above mentioned short wave approximation requires

$$
k^{2} \gg \frac{1}{l_{\min }^{2}} \sim \frac{1}{D t}
$$

Also, the Lewis number

$$
L e=\frac{\kappa}{D} \gg 1 .
$$

This demonstrates that the characteristic length scale related to thermal diffusion is greater than that of the free volume one at a fixed time scale. As shown later, one can notice that these time and length scales will appear and play essential roles again and again, at various stages during shear banding.

The other important issue is that the above parameters constitute one dimensionless number relevant to energy, that is

$$
B=\frac{K P \tau}{\rho C_{v} Q}=\frac{K \tau \gamma}{\rho C_{v} \Delta \theta} \frac{P \Delta \theta}{Q \gamma} .
$$

This number indicates how much the thermal softening $P \Delta \theta$ takes over the strain hardening $Q \gamma$, owing to the temperature rise $\Delta \theta$ resulting from plastic work $\tau$.

We will come back to the implications of these time and length scales as well as dimensionless numbers later.

\section{Comparison of shear banding due to free volume creation and ther- mal softening}

\subsection{Shear instability}

Firstly, the shear instability is examined due to thermal softening and free volume creation, re- 
spectively. Later for simplicity, we use T-instability for that owing to thermal softening and V-instability for that owing to free volume creation. For either T- or V-instability, their corresponding characteristic equations become a cubic equation:

$$
\alpha^{3}+a_{2} \alpha^{2}+a_{1} \alpha+a_{0}=0
$$

Then, in accord with the Routh-Hurwitz criterion for stability, as soon as any of the following three inequalities violates, instability may occur,

$$
\begin{gathered}
a_{2}>0, \\
a_{2} a_{1}-a_{0}>0, \\
a_{0}>0 .
\end{gathered}
$$

For the case of thermally-assisted softening,

$$
\begin{gathered}
a_{2}=\beta / \rho C_{v}=\left(K \dot{\gamma} P+\lambda k^{2}+R C_{v} k^{2}\right) / \rho C_{v}, \\
a_{1}=\omega k^{2} / \rho^{2} C_{v}=k^{2}\left(\lambda R k^{2}+\rho C_{v} Q-K \tau P\right) / \rho^{2} C_{v}, \\
a_{0}=\lambda Q k^{4} / \rho^{2} C_{v} .
\end{gathered}
$$

Though instability depends on a complicated combination of variables, a simple indication of the instability can be deduced at the adiabatic limit $\frac{[25]}{}$ :

$$
K \tau P>\rho C_{V} Q .
$$

This is exactly the requirement of dimensionless number $B>1$. Therefore, T-instability is essentially an energy related one. On the other hand, for the case of free volume creation,

$$
\begin{gathered}
a_{2}=\left(D k^{2}-G_{\xi}\right)+R k^{2} / \rho+G_{\tau} F, \\
a_{1}=k^{2}\left[Q+R\left(D k^{2}-G_{\xi}\right)\right] / \rho, \\
a_{0}=k^{2} Q\left(D k^{2}-G_{\xi}\right) / \rho .
\end{gathered}
$$

One can easily see that the term $\left(D k^{2}-G_{\xi}\right)$ plays a key role in instability, since only this term can change the sign of the coefficients. Clearly, provided $\left(D k^{2}-G_{\xi}\right)$ is negative, instability may occur, i.e.

$$
k^{2}<G_{\xi} / D
$$

So, long wave perturbations $k^{2}<G_{\xi} / D$ always lead to instability. This is very different from thermal softening. Also, this is not energy related, but relevant to the so-called compound creation of free volume $G_{\xi}$. Or say, the compound creation of free volume $G_{\xi}$ (with dimension of inverse time) is the power to motivate the instability, other than the softening owing to free volume $F$. In this aspect, the key role of compound creation of free volume $G_{\xi}$ in V-instability is similar to that in damage localization ${ }^{[26,27]}$. For very long wave perturbation $(k \rightarrow 0)$, even slight compound creation of free volume $G_{\xi}$ can trigger the instability. For instance, for a small test piece $\left(10^{-3} \mathrm{~m}\right)$, as long as $G_{\xi}>$ $D k^{2} \sim 10^{-16} / 10^{-3 \times 2} \sim 10^{-10} \mathrm{~s}^{-1}$, the instability will appear. So, it seems that the free volume creation provides an easier way for instability to occur than thermal softening.

Now we can turn to the coupled case, eqs. (6)-(10). Notice that the coupling effect appears in the term of $\omega\left(D k^{2}-G_{\xi}\right)$ in coefficient $a_{1}$, as the case of thermal softening. However, unlike the case of thermal softening, there is a key term $\left(D k^{2}-G_{\xi}\right)$ in the coefficient $a_{0}$, like the case of free volume creation. Therefore, for the coupled case, the instability may appear more likely as that owing to 
free volume creation. It is noted that, although the momentum and energy equations are incorporated into the govern equation group for the coupled thermal softening and free volume creation problem, whether instability occurs or not is not explicitly related to the thermal softening according to eq. (34). A question naturally arises: Does thermal softening exert no influence on the initiation of shear banding instability? The answer is opposite. Actually, these factors markedly influence instability in the dynamic case $e^{[18]}$.

\subsection{Growth time of shear instability}

As soon as instability occurs, how fast it grows is the other key issue for shear banding. Again, we examine the problem from perturbation approximation to get the estimation of growth time separately.

For T-instability, the approximate growth time can be estimated as ${ }^{[18,25]}$

$$
t_{\text {instability }} \approx 1 / \alpha=\frac{R / Q}{(K P \tau) /\left(\rho C_{v} Q\right)-1}=\frac{t_{R Q}}{B-1} .
$$

Obviously, at an early stage, i.e. $0<B-1 \ll 1$, it grows very slowly. However, provided $B-1$ becomes $O(1)$, the growth will accelerate to the order of $t_{R Q}$, namely the relaxation time scale.

On the other hand, for $\mathrm{V}$-instability, the instability growth would be

$$
t_{\text {instability }} \approx 1 / \alpha=t_{G}=1 / G_{\xi} .
$$

So, the growths in the two instabilities are quite different. From the data in Table 1, the corresponding time might be $t_{G} \sim 10^{0} \mathrm{~s}$. This seems to be able to compare with the time scale $t_{\mathrm{ex}} \sim 10^{1} \mathrm{~s}$ for static tests, but too long for dynamic tests with time scale $t_{\mathrm{ex}} \sim 10^{-5} \mathrm{~s}$. If so, combined with the difference of instability occurrence, a probable picture of shear instability might be as follows. At first, compound free volume creation triggers the instability and governs its early stage. Afterward, shear banding may be governed by free volume creation, provided the loading time is long enough in comparison with the intrinsic time scale $t_{G}$ (like static loading) and there is still not enough plastic strain to trigger thermal instability. Or it may appear as a thermo-assisted one at a later stage, once thermal softening can accelerate instability growth with the increase of plastic deformation, especially for the short loading time ( $\mu \mathrm{s}-\mathrm{ms}$ ) under impact (see eq. (35)). Certainly, the most uncertain issue involved in the estimation is the magnitude of the compound free volume creation $G(\xi)$. Now, we turn to bandwidth estimate, to see if there is a further verification of the picture.

\subsection{Width of shear bands}

Since most experimental observations are post-mortem patterns of shear bands, it is useful to get some estimation on mature shear bandwidth and to make comparison to the observations. Based on basic eqs. (1)-(4), the late stage steady equations and band-like solutions for the two cases are (to facilitate comparison, the following two columns corresponding to thermal softening and free volume creation, respectively):

T-banding:

$$
\begin{gathered}
\lambda \frac{\partial^{2} \theta}{\partial y^{2}}+K \tau \dot{\gamma}=0, \\
\dot{\gamma}=g(\tau, \theta), \quad \frac{\partial \tau}{\partial y}=0,
\end{gathered}
$$




$$
y=\sqrt{\frac{\lambda}{2 K \tau}} \int_{\theta}^{\theta_{m}} \frac{\mathrm{d} \varsigma}{\sqrt{\int_{\varsigma}^{\theta_{m}} g(\tau, \eta) \mathrm{d} \eta}}
$$

V-banding:

$$
\begin{gathered}
D \frac{\partial^{2} \xi}{\partial y^{2}}+G(\xi, \theta, \tau)=0, \\
\frac{\partial \tau}{\partial y}=0, \\
y=\sqrt{\frac{D}{2}} \int_{\varsigma}^{\xi_{m}} \frac{\mathrm{d} \varsigma}{\sqrt{\int_{\varsigma}^{\xi_{m}} G(\eta, \theta, \tau) \mathrm{d} \eta}},
\end{gathered}
$$

where $\lambda=\rho C_{v} \kappa$ is thermal conductivity, $y$ is the coordinate vertical to the band and subscript $m$ denotes the value at the center of the band. Clearly, there is a similarity between thermal and free volume diffusions in the steady solutions. However, the difference is distinctive: Their integrands are different. One is the strain rate, namely external time scale, and the other is the free volume creation $G$, namely an intrinsic one. Then an oversimplified but simple expression of the shear bandwidth in the two cases could be written as

$$
\begin{gathered}
\delta_{\mathrm{T}} \sim \sqrt{\kappa \frac{\gamma^{*}}{\dot{\gamma}^{*}} \frac{\rho C_{v} \theta^{*}}{K \tau} \tau^{*}} \sim \sqrt{\kappa \frac{\gamma^{*}}{\dot{\gamma}^{*}}}, \\
\delta_{\mathrm{V}} \sim \sqrt{D \frac{\xi^{*}}{G^{*}}},
\end{gathered}
$$

where $*$ denotes the values within the band. From the data available in Table $1, \delta_{\mathrm{T}} \sim \mu \mathrm{m}$ and $\delta_{\mathrm{V}}$ $\sim \mathrm{nm}$, respectively. The existing experimental results have shown that the shear bandwidth in BMGs is $\leqslant 10-20 \mathrm{~nm}^{[28]}$. Obviously, the thermal shear band width is much larger than the experimentally measured shear bandwidth. But the shear band resulting from free volume creation can match the experimental results well. The comparison between $\delta_{\mathrm{T}}$ and $\delta_{\mathrm{V}}$ demonstrates that free volume creation may be the key factor to shear banding in BMGs. Note that $\delta_{\mathrm{T}} / \delta_{\mathrm{V}}$ is quite relevant to Lewis number, except involving different time scales for different diffusions, $\gamma / \dot{\gamma}$ and $\xi / G$, respectively. For the coupled case, the instability may appear more likely as that owing to free volume creation, but thermal softening does exert influence on the initiation of adiabatic shear banding instability. The shear bandwidth may lie between $\delta_{\mathrm{T}}$ and $\delta_{\mathrm{V}}$, i.e. $\delta_{\mathrm{T}}>\delta_{\mathrm{T}+\mathrm{V}}>\delta_{\mathrm{V}}$.

\subsection{Comparisons of the calculated shear bandwidth}

Whether is it thermal softening or free volume creation plays a key role on shear banding in BMGs? Although the preliminary confirmation from the instability criterion demonstrates that, as the compound free volume creation over its diffusion, the shear instability can occur and lead to shear banding, the existing experimental methods cannot measure those key parameters related to shear banding now, especially for $G$. Only the shear bandwidth and qualitatively increased free volumes in the mature shear bands can be observed by HRTEM. Therefore, numerical computations have become a valuable mean to study shear banding in BMGs. The closely related picture of shear banding could be deduced by the comparison between numerical and experimental re- 
sults. Based on the governing eqs. (1) - (4) for one-dimensional simple shear of BMGs, the shear banding is computed under thermal softening, free volume creation and coupled thermal and free volume creation-softening, respectively.

The initial value of free volume concentration is set and its evolution is ignored in the computation of the thermal softening case. Oppositely, the temperature keeps constant and the evolution of free volume concentration must be accounted in the free volume creation case. In the coupled thermal and free volume creation-softening case, the evolutions of both temperature and free volume concentration are computed. The computation results for the three cases are given in Figure 1. Clearly, the mature shear bands under these cases have their own apparent characteristics.

Firstly, the shear band resulting from thermal softening is much wider than that resulting from free volume creation or the coupled case. Among these results, the width of shear band resulting from free volume creation is almost equal to that from the coupled case, i.e. $w \approx 10 l_{D} \sim 10 \mathrm{~nm}$. This is closely equal to the experimental observations ${ }^{[28]}$. However, the width of shear band resulting from thermal softening is $w \approx 250 l_{D} \sim 250 \mathrm{~nm}$ and is much larger than that from the experimental observations. The significant difference among T-shear band, V-shear band and experimental results indicates that free volume creation is the key factor to shear banding in BMGs, not the thermal softening. The fact that the shear strain in the shear band resulting from the coupled case is larger than that from free volume creation demonstrates that adiabatic heating promotes the formation of shear bands in BMGs. Secondly, the shear strain in the shear band resulting from free volume creation is larger than that from thermal softening at the same whole deformation, demonstrating that free volume creation-softening is more intensive than thermal softening, and will easily lead to damage or failure of BMGs. This tendency may be one of the reasons leading to brittle fracture of BMGs.

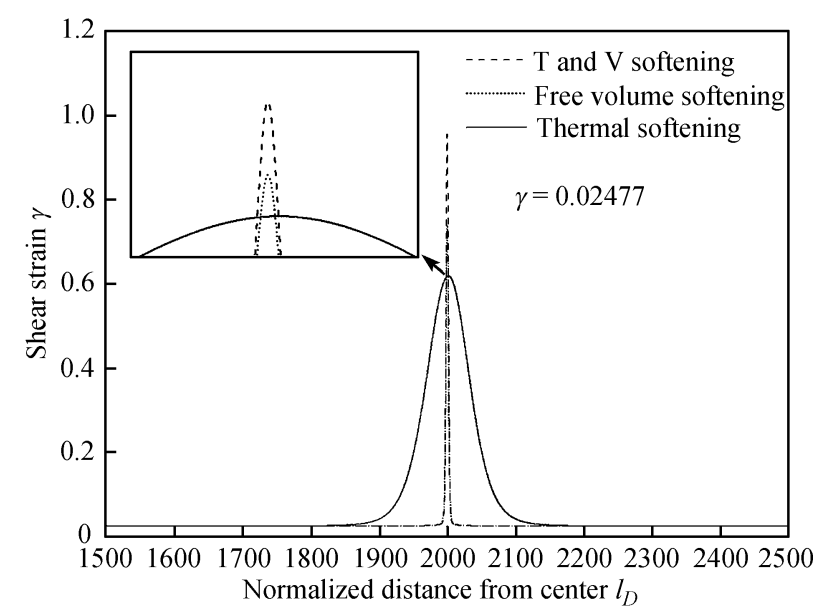

Figure 1 The computed results under thermal softening, free volume creation and coupled thermal and free volume creation-softening.

\section{Discussion}

The main differences of the two mechanisms of shear banding, i.e. thermo-softening and free volume creation, can be summarized as follows: 
(1) Thermal assisted shear instability and its growth rate are governed by thermal softening and are energy related, i.e. $B>1$; but the free volume instability is governed by the compound free volume creation $G_{\xi}>D k^{2}$ and not energy related. This implies that the perturbations with long wave length $k^{2}<G_{\xi} / D$ will always lead to instability. So, V-instability may occur more easily than T-instability. Accordingly, the $\mathrm{T} \& \mathrm{~V}$ coupled instability may appear more like V-instability. The analysis is different from Huang et al.'s model ${ }^{[8]}$ which only focuses on quasi-static deformation and ignores temperature effects. So, their model can hardly provide the comparative understanding of shear banding with various time scales and temperature effects. Furthermore, considerable research works have demonstrated that the formation of shear bands in BMGs is a rate-dependent process $21,22,29-33]$. Especially, the more obvious melting characteristics on the fracture surfaces in dynamic test than in the quasi-static case on BMGs ${ }^{[20-22]}$ demonstrate the difference of temperature effect on the shear banding between static and dynamic cases. So, the coupled and comparative analysis, provided in this paper, on the shear banding with both adiabatic hearting and free-volume creations in BMGs is of scientific significance.

(2) There are various characteristic time scales. Importantly, V-instability grows with its own “clock”, i.e. $t_{G}=1 / G_{\xi}$; whereas T-instability grows with the relaxation time regulated by how far beyond instability, $t_{R Q} /(B-1)$. So, the Deborah number $D e_{G}=\frac{t_{G}}{t_{\text {ex }}} \sim \frac{\dot{\gamma}}{G_{\xi} \gamma} \sim \begin{cases}\sim<(1), & \text { dynamic } \\ \sim O(1), & \text { static }\end{cases}$ may be a measure of the observability of V-instability.

To briefly sum up the above results on shear instability and its growth, Figure 2 gives a sketch. For simplicity, it shows situations under long wave assumption, also adiabatic assumption only. For the cases with $B \gg 1$, shear band would appear more probably by thermal softening (the upper part of the sketch). On the other hand, only $D e_{G} \sim<1$ can allow V-instability to grow to become visible during the loading time (left down corner of the sketch), though V-instability can occur on the whole plane of the sketch under long wave assumption.

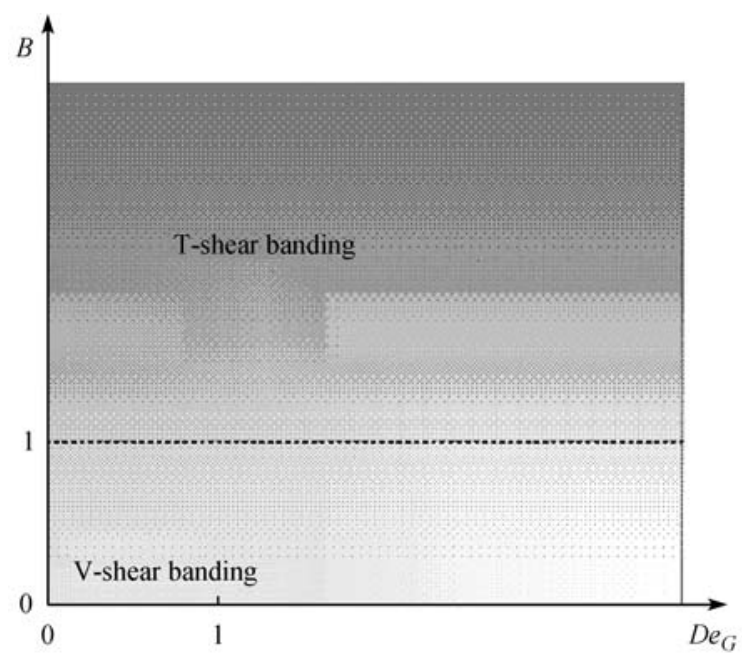

Figure 2 A sketch of shear banding owing to thermal softening (characterized by $B$ ) and/or compound free volume creation (characterized by $D e_{G}$ ), under long wave assumption (also adiabatic assumption). The dashed line indicates the threshold for T-instability, i.e. $B>1$; whereas V-instability $\left(D e_{G}>0\right)$ does not need such a threshold. The two partially overlapped darker regions, denoting the growth fast enough to be visible, imply the two probable types of shear banding. 
(3) The mature shear bandwidth $\delta$ is governed by corresponding diffusions within the band: thermal and free volume diffusions, $\delta_{\mathrm{T}} \sim \sqrt{\kappa \frac{\gamma^{*}}{\dot{\gamma}^{*}}}$ and $\delta_{\mathrm{V}} \sim \sqrt{D \frac{\xi^{*}}{G^{*}}}$. Note that the time scales are different: The former depends on external time, and the latter on its own time scale $\xi / G$. Though the time difference is significant, as a rough guide, Lewis number $L e=\frac{\kappa}{D} \gg 1$ implies the great difference of the band width.

After taking the magnitudes of various parameters and variables of BMGs into account, a probable road map of shear banding in BMGs can be shown in Table 2.

Table 2 A possible road map of shear banding in BMGs

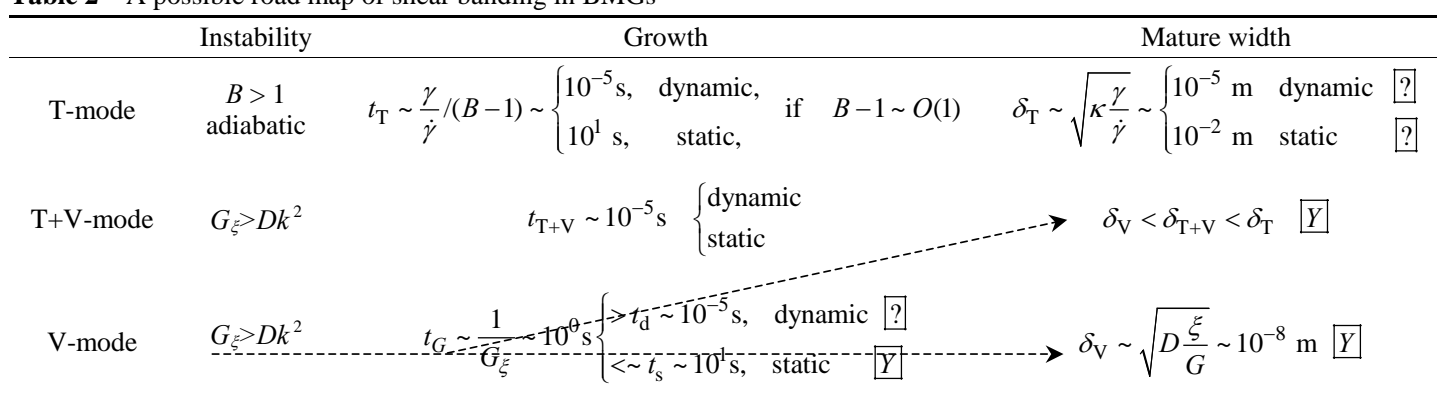

$Y$ observable; ? hardly observable.

(4) The computed results demonstrate that the width of shear band resulting from thermal softening is much larger than that of experimental observations. The significant difference between T-shear banding and experimental results indicates that the thermal softening is not the key factor to shear banding. While the width of shear band resulting from free volume creation is closely consistent with the experimental observations. The consistence of shear band width demonstrates that free volume creation is the key factor to shear banding. The fact that the shear strain in the shear band under coupled thermal and free volume creation-softening is larger than that under free volume creation demonstrates that adiabatic heating promotes the formation of shear bands in BMGs.

(5) As the article mentioned, the most uncertain issue involved in the estimation is the magnitude of the compound free volume creation $G(\xi)$. For our analysis, only a general form of free volume creation $G$ is adopted, with its magnitude and derivative. At present, the phenomenological model on $G$ is based on Spaepen et al.'s works ${ }^{[5,7,8]}$ and the detailed form and its elaborations could be found in ref. [5]. Although the free volume model developed by Spaepen et al. was a phenomenological model, it interpreted some experimental results successfully ${ }^{[34-38]}$. Later, Argon ${ }^{[6]}$ argued that the plastic deformation of metallic glasses was the result of thermally activated shear transformations around free volume regions under an applied shear stress. In view of the spirit that any satisfactory theory of plasticity must include dynamical state variables beyond stress and strain, Langer and his coworkers proposed a shear-transformation-zone (STZ) theory of deformation in metallic glass recently ${ }^{[39]}$. One of the most fundamental differences between the previous work ${ }^{[5-7]}$ and the STZ theory is that the system is effectively at zero temperature. The transition between one state and the other constitutes an elementary increment of shear strain and controls the mechanical 
properties in BMGs. But, the evolvement of free volume related to the transition is not clearly clarified either. Indeed, we do not concentrate our interest on the detailed value of $G$. These rough estimates do not affect the methods proposed by this article to study the formation mechanism of shear band in BMGs. We just propose an approach, a new idea.

Above all, shortly but not accurately, the shear instability resulting from compound free volume creation may occur easily but maybe grows slowly and the band looks narrow. Whereas the thermal assisted shear instability can occur only after an energy related criterion $(B>1$ under adiabatic approximation) is met, but its growth can be accelerated to match the external loading and the band looks wider than the former. Therefore, for the coupled case, shear instability almost always starts with compound free volume creation (the left part of the lower dashed line in Table 2 ), and it may grow in the same manner provided its Deborah number $D e_{G} \sim<1$, namely under static loading (see the lower dashed line in Table 2). But it may also grow and mature in a coupled thermal and free volume creation assisted manner more likely under impact loading (the branched upper dashed line in Table 2).

As mentioned previously, the most uncertain issue is the magnitude of the compound free volume creation $G(\xi)$, which needs further comprehensive examination.

\section{Summaries}

Comparative studies of shear banding resulting from thermal softening, free volume creation and coupled thermal and free volume creation-softening are performed to seek the instability criterions, growth time and characteristics of shear bands in BMGs in the present study. The results can be summarized as follows:

(1) The shear instability of BMGs resulting from thermal softening, free volume creation and coupled thermal and free volume creation-softening are studied by a linear stability analysis. Criteria of shear instability of BMGs for the aforementioned cases are achieved.

(2) A series of governing parameters and temporal-spatial scales governing the shear band formation in BMGs are obtained.

(3) The relationship between nondimensional Deborah number and the T-shear banding or V-shear banding is revealed. Furthermore, a sketch of shear banding owing to thermal softening and/or compound free volume creation under long wave assumption is proposed.

(4) Comparative studies among experimental observations, analysis and computed results demonstrate that free volume creation plays a dominant role in the formation of shear bands, and adiabatic heating softening exerts a secondary influence at dynamic tests in BMGs.

1 Inoue A, Zhang T, Masumoto T, et al. La-Al-Ni amorphous alloys with a wide supercooled liquid region. Mater Trans JIM, 1989, 30: 965-972

2 Johnson W L. Bulk glass-forming alloys: Science and technology. MRS Bull, 1999, 24: 42 - 56

3 Wang W H, Dong C, Shek C H. Bulk metallic glasses. Mater Sci \& Eng R, 2004, 44: 45-89[DOI]

4 Ashby M F, Greer A L. Metallic glasses as structural materials. Scripta Mater, 2006, 54: 321 - 326[DOI]

5 Spaepen F. A microscopic mechanism for steady state inhomogeneous flow in metallic glasses. Acta Metall, 1977, 25: 407415[DOI]

6 Argon A. Plastic deformation in metallic glasses. Acta Metall, 1979, 27: 47 - 58[DOI]

7 Steif P S, Spaepen F, Hutchinson J W. Strain localization in amorphous metals. Acta Metall, 1982, 30: 447-455[DOI]

8 Huang R, Suo Z, Prevost J H, et al. Inhomogeneous deformation in metallic glasses. J Mech Phys Solids, 2002, 50: 10111027[DOI] 
9 Leamy H J, Chen H S, Wang T T. Plastic flow and fracture of metallic glass. Metall Trans, 1972, 3: 699-708[DOI]

10 Liu C T, Heatherly L, Easton D S, et al. Test environments and mechanical properties of Zr-base bulk amorphous Alloys. Metall Mater Trans A, 1998, 29: 1811-1820[DOI]

11 Pampillo C A. Localized shear deformation in a glassy metal. Scripta Metall, 1972, 6: 915-918[DOI]

12 Krishnanand K D, Cahn R W. Recovery from plastic deformation in a Ni/Nb alloy glass. Scripta Metall, 1975, 9: 12591261[DOI]

13 Bruck H A, Rosakis A J, Johnson W L. The dynamic compressive behavior of beryllium bearing bulk metallic glasses. J Mater Res, 1996, 11: 503-511[DOI]

14 Hufnagel T C, Jiao T, Li Y, et al. Deformation and failure of $\mathrm{Zr}_{57} \mathrm{Ti}_{5} \mathrm{Cu}_{20} \mathrm{Ni}_{8} \mathrm{Al}_{10}$ bulk metallic glass under quasi-static and dynamic compression. J Mater Res, 2002, 17: 1441-1445[DOI]

15 Lewandowski J J, Greer A L. Temperature rise at shear bands in metallic glasses. Nature Mater, 2006, 5: 15-18[DOI]

16 Spaepen F. Must shear band be hot? Nat Mater, 2006, 5: 7-8[DOI]

17 Falk M L, Shi Y F. Strain localization in a molecular-dynamics model of a metallic glass. In: Supercooled Liquids, Glass Transition and Bulk Metallic Glasses, Vol. 754. Pittsburgh: Mat Res Soc, 2003. 1

18 Dai L H, Yan M, Liu L F, et al. Adiabatic shear banding instability in bulk metallic glasses. Appl Phys Lett, 2005, 87: 141916-1-3[DOI]

19 Davison L, Stevens A L. Continuum measures of spall damage. J Appl Phys, 1972, 43: 988 - 994[DOI]

20 Conner R D, Dandliker R B, Scruggs V, et al. Dynamic deformation behavior of W-fiber/metallic-glass matrix composites. Int J Impact Eng, 2000, 24: 435-444[DOI]

21 Liu L F, Dai L H, Bai Y L, et al. Characterization of rate-dependent shear behavior of Zr-based bulk metallic glass using shear-punch testing. J Mater Res, 2005, 21: 153-160[DOI]

22 Liu L F, Dai L H, Bai Y L, et al. Initiation and propagation of shear bands in Zr-based bulk metallic glass under quasi-static and dynamic loadings. J Non-Crystall Solids, 2005, 351: 3259-3270[DOI]

23 Liu L F, Dai L H, Bai Y L, et al. Behavior of multiple shear bands in Zr-based bulk metallic glass. Mater Chem Phys, 2005, 93: $174-177[\mathrm{DOI}]$

24 Bai Y, Dodd B. Adiabatic Shear Localization: Occurrence, Theories and Applications. Oxford: Pergamon Press, 1992

25 Bai Y L. Thermo-plastic instability in simple shear. J Mech Phys Solids, 1982, 30: 195-207[DOI]

26 Bai Y L, Xia M F, Ke F J, et al. Damage field equation and criterion for damage localization, in rheology of bodies with Defects. In: Wang R, ed. Proc of IUTAM Symposium. Dordrecht: Kluwer, 1998. 55

27 Bai Y L, Bai J, Li H L, et al. Damage evolution, localization and failure of solids subjected to impact loading. Int J Impact Eng, 2000, 24: 685-701[DOI]

28 Zhang Y, Greer A L. Thickness of shear bands in metallic glasses. Appl Phys Lett, 2006, 89: 071907-1-3[DOI]

29 Mukai T, Nieh T G, Kawamura Y, et al. Dynamic response of a $\mathrm{Pd}_{40} \mathrm{Ni}_{40} \mathrm{P}_{20}$ bulk metallic glass in tension. Scripta Mater, 2002, 46: $43-47[\mathrm{DOI}]$

30 Schuh C A, Nieh T G. A survey of instrumented indentation studies on metallic glasses. J Mater Res, 2004, 19: 46-57

31 Jiang W H, Atzmon M. Rate dependence of serrated flow in a metallic glass. J Mater Res, 2003, 18: 755-757[DOI]

32 Dai L H, Liu L F, Yan M, et al. Serrated plastic flow in a Zr-based bulk metallic glass during nanoindentation. Chin Phys Lett, 2004, 21: 1593-1594[DOI]

33 Liu L F, Dai L H, Bai Y L, et al. Strain rate-dependent compressive deformation behavior of Nd-based bulk metallic glass. Intermetallics, 2005, 13: 827-832[DOI]

34 Donvan P E, Stobbs W M. The structure of shear bands in metallic glass. Acta Metall, 1981, 29: 1419-1436[DOI]

35 Pekarskaya E, Kim C P, Johnson W L. In-situ transmission electron microscopy studies of shear bands in a bulk metallic glass based composite. J Mater Res, 2001, 16: 2513-2518[DOI]

$36 \mathrm{Li} \mathrm{J}$, Wang Z L, Hufnagel T C. Characterization of nanometer-scale defects in metallic glasses by quantitative high-resolution transmission electron microscopy. Phys Rev B, 2002, 65: 144201-1-6[DOI]

37 Flores K M, Schuh D, Dauskardt R H, et al. Characterization of free volume in a bulk metallic glass using positron annihilation spectroscopy. J Mater Res, 2002, 17: 1153-1161[DOI]

$38 \mathrm{Lu}$ J, Ravichandran G. Pressure dependent flow behavior of $\mathrm{Zr}_{41.2} \mathrm{Ti}_{13.8} \mathrm{Cu}_{12.5} \mathrm{Ni}_{10} \mathrm{Be}_{22.5}$ bulk metallic glass. J Mater Res, 2003, 18: 2039-2049[DOI]

39 Falk M L, Langer J S. Dynamics of viscoplastic deformation in amorphous solids. Phys Rev E, 1998, 57: 7192-7205[DOI] 